\title{
Detection and Memory of Non-Racemic Molecules by a Racemic Host Polymer Film
}

Anna Maria Buono, Ivano Immediata, Paola Rizzo, Gaetano Guerra* Dipartimento di Chimica, Università di Salerno Via Ponte don Melillo, Fisciano 84084 Salerno (Italy)

\section{Supporting information}

The s-PS used in this study was manufactured by Dow Chemical Company under the trademark Questra 101. The ${ }^{13} \mathrm{C}$ nuclear magnetic resonance characterization showed that the content of syndiotactic triads was over $98 \%$. The weight-average molar mass obtained by gel permeation chromatography (GPC) in trichlorobenzene at $135^{\circ} \mathrm{C}$ was found to be $\mathrm{M}_{\mathrm{w}}=3.2 \times 10^{5}$ with the polydispersity index, $M_{w} / M_{n}=3.9$.

s-PS films for the CD measurements, having thickness in the range $0,1-0,2 \mu \mathrm{m}$, have been prepared by spin-coating onto quartz surface of a $0.25 \mathrm{wt} \%$ chloroform solution.

CD spectra were measured using a Jasco J-715 spectropolarimeter. All measurements were performed using the following parameters: single scan, continuous scanning mode (350-190 nm range), $200 \mathrm{~nm} / \mathrm{min}$ scanning speed, $2 \mathrm{~nm}$ SBW (constant bandpass mode), $0.2 \mathrm{~nm}$ data interval, vertical scale in autoranging mode, no baseline correction.

Wide-angle X-ray diffraction (WAXD) patterns with nickel filtered CuK $\alpha$ radiation were obtained, in reflection, with an automatic Philips powder diffractometer.

The surface analyses were performed by using an AFM TMX 2100 Explorer Microscope (Veeco). The AFM micrographs were obtained by operating in non contact mode.

The possible ability of a given molecule to be guest of a molecular complex phase with s-PS has been also explored, already for low guest content, by linear dichroism measurements on uniaxially oriented $\delta$ form films. ${ }^{30,31}$ Fourier-transform infrared (FTIR) spectra (not shown) of uniaxially stretched $\delta$-form s-PS films with the chiral molecules of Scheme 1 (from $1 \mathrm{wt} \%$ up to 10 wt\%) present highly dichroic solute vibrational peaks, clearly confirming that these molecule are guest of the crystalline polymeric host phase.

Infrared spectra were obtained at a resolution of $2.0 \mathrm{~cm}^{-1}$ with a Vector 22 Bruker spectrometer equipped with deuterated triglycine sulphate (DTGS) detector and a Ge/KBr beam splitter. The frequency scale was internally calibrated to $0.01 \mathrm{~cm}^{-1}$ using a He-Ne laser. 32 scans were signal averaged to reduce the noise. Polarized infrared spectra $w$ ere recorded by use of a SPECAC 12000 wire grid polarizer.

30 A. R. Albunia, S. Di Masi, P. Rizzo, G. Milano, P. Musto, G. Guerra, Macromolecules 2003, 36, 8695-8703.

31 A. R. Albunia, G. Milano, V. Venditto, G. Guerra, J. Am. Chem. Soc. 2005, 127, 13114-13115. 